\title{
Article \\ Search for Sub-Solar Mass Binaries with Einstein Telescope and Cosmic Explorer
}

\author{
Rafael C. Nunes $\mathbb{D}$
}

Divisão de Astrofísica, Instituto Nacional de Pesquisas Espaciais, Avenida dos Astronautas 1758, São José dos Campos 12227-010, SP, Brazil; rafadcnunes@gmail.com

\begin{abstract}
A possible detection of sub-solar mass ultra-compact objects would lead to new perspectives on the existence of black holes that are not of astrophysical origin and/or pertain to formation scenarios of exotic ultra-compact objects. Both possibilities open new perspectives for better understanding of our universe. In this work, we investigate the significance of detection of sub-solar mass binaries with components mass in the range: $10^{-2} M_{\odot}$ up to $1 M_{\odot}$, within the expected sensitivity of the ground-based gravitational waves detectors of third generation, viz., the Einstein Telescope (ET) and the Cosmic Explorer (CE). Assuming a minimum of amplitude signal-to-noise ratio for detection, viz., $\rho=8$, we find that the maximum horizon distances for an ultra-compact binary system with components mass $10^{-2} M_{\odot}$ and $1 M_{\odot}$ are $40 \mathrm{Mpc}$ and $1.89 \mathrm{Gpc}$, respectively, for ET, and $125 \mathrm{Mpc}$ and $5.8 \mathrm{Gpc}$, respectively, for $\mathrm{CE}$. Other cases are also presented in the text. We derive the merger rate and discuss consequences on the abundances of primordial black hole $(\mathrm{PBH}), f_{\mathrm{PBH}}$. Considering the entire mass range $\left[10^{-2}-1\right] M_{\odot}$, we find $f_{\mathrm{PBH}}<0.70(<0.06)$ for $\mathrm{ET}(\mathrm{CE})$, respectively.
\end{abstract}

Keywords: gravitational wave astronomy; ground-based detectors; primordial black hole

check for updates

Citation: Nunes, R.C. Search for Sub-Solar Mass Binaries with Einstein Telescope and Cosmic Explorer. Entropy 2022, 24, 262. https://doi.org/10.3390/e24020262

Academic Editor: Luis Alfredo Herrera Cometta

Received: 22 December 2021 Accepted: 13 January 2022 Published: 11 February 2022

Publisher's Note: MDPI stays neutral with regard to jurisdictional claims in published maps and institutional affiliations.

Copyright: (c) 2022 by the author. Licensee MDPI, Basel, Switzerland. This article is an open access article distributed under the terms and conditions of the Creative Commons Attribution (CC BY) license (https:// creativecommons.org/licenses/by/ $4.0 /)$.

\section{Introduction}

We are in the beginning of the era of gravitational wave (GWs) astronomy. The LIGO/ VIRGO observatories already detected more than 50 coalescing compact binaries events [1,2], and the probes have targeted binary systems with total masses in the range [2-600] $M_{\odot}[3,4]$. The LIGO and Virgo detectors are also sensitive to ultra-compact binaries with components below $1 M_{\odot}$, as the compactness is close to that of the black holes. In the probes for GWs from the coalescence of sub-solar mass binaries, recently performed in [5-11], no convincing candidates were found in LIGO/VIRGO data.

Search for the sub-solar mass ultra compact binaries is worthwhile because it may provide direct evidence of the existence of black holes that are not of astrophysical origin or formation of exotic ultra-compact objects. We know that in the standard stellar evolution models, the lightest compact objects are formed when stellar remnants exceed the Chandrasekhar mass limit $\sim 1.4 M_{\odot}$. Beyond the Chandrasekhar mass limit, the electron degeneracy pressure in the star's core is insufficient to balance the star's own gravitational self-attraction, and, therefore, can no longer prevent the gravitational collapse of a white dwarf. The lightest remnants that exceed the Chandrasekhar mass limit will form neutron stars, and when the neutron degeneracy pressure cannot prevent collapse, heavier stellar remnants will collapse to form black holes. To the present knowledge, there is no model for forming neutron stars for $<1 M_{\odot}$. On the other hand, black holes appear to have a minimum mass $\sim 5 M_{\odot}$. Furthermore, the observations confirm that there is a gap $\sim[2,5] M_{\odot}$ between the neutron star and black hole masses [12-14]. Thus, detecting ultra-compact objects below $1 M_{\odot}$ could challenge the stellar evolution or possibly hint at some unconventional formation scenarios for such objects.

The theoretical postulations for the existence of alternative channels for the formation of black hole were proposed 50 years ago [15-19]. The main motivation is that black holes could have formed in the early universe through the collapse of highly over-dense regions, 
the so-called primordial black holes (PBHs). It has been shown that PBHs can also form at late times [20,21]. If PBHs exist, these can naturally account for the dark matter or at least explain a fraction of the dark matter abundance [22-24]. Various studies using the observations of black hole mergers by LIGO/Virgo data are carried out to constrain the PBHs and their abundance [25-36], including proposals on how to distinguish a PBH from an astrophysical one $[37,38]$. Furthermore, several analyses and theoretical calculations are carried out to investigate PHBs for the prospects of future GWs detectors, such as LISA [39-41] and Einstein telescope (ET) [42,43]. See the work in [44] for a general review on the PBHs. On the other hand, various proposals for non-baryonic dark matter models can produce subsolar mass black holes, as well as possibilities for the formation of some exotic ultra-compact objects, with masses below $1 M_{\odot}$ [45]. Thus, the detection of sub-solar mass ultracompact objects would provide the cleanest signature of such scenarios.

The aim of this work is to search for the possible imprints of sub-solar mass binaries within the expected sensitivity of Einstein Telescope (ET) and Cosmic Explorer (CE). Both instruments are ground-based GWs detectors of third generation, which could be operating in the mid 2030s. With ET and CE, we will be able to determine the nature of the densest matter in the universe, reveal the universe's binary black hole and neutron star populations throughout cosmic time, provide an independent probe of the history of the expanding universe, physics near the black hole horizon, test exotic compact objects, as well as many other questions in fundamental physics and cosmology. See the works in [46-48] for a presentation of scientific objectives with these observatories. In this paper, we show that ET and CE will be able to detect strong signals coming from sub-solar mass binaries system candidate with components mass in the range $\in\left[10^{-2}-1.0\right] M_{\odot}$. Estimating the merger rate of these compact binaries, we discuss consequences on the $\mathrm{PBHs}$ ' abundance.

This paper is structured as follows. In next section, we define the essential quantities to analyze the GWs signals. In Section 3, we present our main results and lastly, in Section 4, we outline our final considerations and perspectives.

\section{Analysis Strategy}

In this section, we briefly summarize the methodology and main information used to search for compact binary system. For a given GW strain signal $h(t)=A(t) \cos [\Phi(t)]$, one can use the stationary-phase approximation for the orbital phase of inspiraling binary system to obtain its Fourier transform $\tilde{h}(f)$. In the case of a coalescing binary system, we have

$$
\tilde{h}(f)=Q \mathcal{A} f^{-7 / 6} e^{i \Phi(f)},
$$

where $\mathcal{A}$ is the GW amplitude computed perturbatively within the so-called post-Newtonian formalism (PN), and can be written as

$$
\mathcal{A}=\sqrt{\frac{5}{96}} \frac{\mathcal{M}_{c}^{5 / 6}}{\pi^{2 / 3} d_{L}}\left(\sum_{i=0}^{6} A_{i}(\pi f)^{i / 3}\right) .
$$

Here $d_{L}$ is the luminosity distance, and the function $Q$ is given by

$$
Q^{2}=F_{+}^{2}\left(1+\cos ^{2}(\iota)\right)^{2}+2 F_{\times}^{2} \cos ^{2}(\iota),
$$

where $\iota$ is the inclination angle of the binary orbital angular momentum with respect to the line of sight, and $F_{+}^{2}, F_{\times}^{2}$ are the pattern functions (specific functions for each detector).

In Equation (1), the function $\Phi(f)$ is the inspiral phase of the binary system:

$$
\Phi(f)=2 \pi f t_{c}-\phi_{c}-\frac{\pi}{4}+\frac{3}{128 \eta v^{5}}\left[1+\sum_{i=2}^{7} \alpha_{i} v^{i}\right],
$$

where the coefficients $\alpha_{i}$ are computed perturbatively in a post-Newtonian formalism.

In this work, we will use the TaylorF2 waveform model, which uses the stationary phase approximation for the waveform, and the 3.5 PN expression for the orbital phase 
of inspiraling binary black holes with aligned spins, along with the tidal effects on the phase (up to the 6PN level) for compact objects. In the above equation, we have defined $v \equiv(\pi M f)^{1 / 3}, M \equiv m_{1}+m_{2}, \eta \equiv m_{1} m_{2} /\left(m_{1}+m_{2}\right)^{2}$, and $\mathcal{M}_{c} \equiv M \eta^{3 / 5}$ to be the inspiral reduced frequency, total mass, symmetric mass ratio, and the chirp mass, respectively. The quantities $t_{c}$ and $\phi_{c}$ are the time and phase of coalescence, respectively.

The amplitude of signal-to-noise ratio (SNR), $\rho$, for a deterministic signal $\tilde{h}(f)$ is given by

$$
\rho^{2} \equiv 4 \operatorname{Re} \int_{f_{\text {low }}}^{f_{u p p e r}} \frac{|\tilde{h}(f)|^{2}}{S_{n}} d f,
$$

where $S_{n}(f)$ is the detector spectral noise density.

Sensitivity curves: We considered the ET and CE power spectral density noises. Both instruments are third-generation ground detectors, covering the frequency range $1-10^{4} \mathrm{~Hz}$. The signal amplitude sensitivity of ET and CE is expected to be more than ten times larger than the current advanced ground-based detectors. For ET, we consider the ET-D sensitivity curve $[46,49,50]$. For $\mathrm{CE}$, we also consider the amplitude spectrum of the detector noise also publicly available in $[47,51]$.

Figure 1 shows the characteristic strain for a qualitative example considering a subsolar mass binary system with components mass $1 M_{\odot}$ at $d_{L}=100 \mathrm{Mpc}$, along with the noise power spectral density for ET, CE and aLIGO. In all the results presented in this work, we use the integration approach on the average over all possible directions and inclinations.

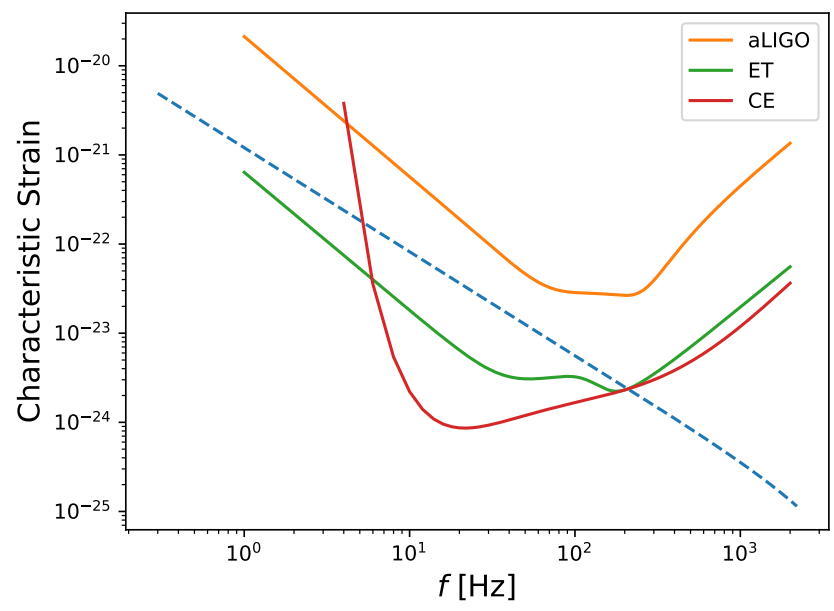

Figure 1. Characteristic strain of a possible sub-solar mass binary system candidate with components mass $1 M_{\odot}$ at $d_{L}=100 \mathrm{Mpc}$ plotted along with $\sqrt{f S_{n}(f)}$, where $S_{n}(f)$ is the noise power spectral density for ET, CE and aLIGO.

Very low mass systems are expected to emit GWs in the frequency range of the groundbased detectors. To understand it, to leading post-Newtonian order, the frequency as a function of time is given by $f(t)=\frac{1}{8 \pi G M_{c} / c^{3}}\left(\frac{5 G M_{c} / c^{3}}{t-t_{c}}\right)^{3 / 8}$. For example, an equal mass binary with components masses $1 M_{\odot}, 0.2 M_{\odot}$, will have GW frequencies of $0.25 \mathrm{~Hz}$ and $0.70 \mathrm{~Hz}$, respectively, one year prior to merger. The maximum frequency can be determined by the frequency of the innermost stable circular orbit $\left(f_{\mathrm{ISCO}}\right)$, where $f_{\mathrm{ISCO}}=\frac{c^{3}}{6 \sqrt{6} \pi G M}$. For binary system with components masses $1 M_{\odot}, 0.2 M_{\odot}$, we have $f_{\text {ISCO }}=2200 \mathrm{~Hz}$, $10,100 \mathrm{~Hz}$, respectively. Thus, sub-solar mass binaries compact inspiraling can be visible at the maximum frequency range sensitivity of the ground-based detectors before the merger. On the other hand, even considering several years prior to merger, the expected GW amplitude will be beyond and below the LISA band operation. These qualitative aspects are clear in Figure 1. We assume that the coalescence of sub-solar mass black hole binaries have negligible spin. This is consistent with the predictions of spin distributions presented in [21,52-54]. 
Merger rate for null results: We can calculate the maximum distance for which an optimally located and oriented source would be observed with some $\rho$ value. In general, the detectors will measure a weaker response to GWs, depending on the location and orientation of the binary system. This reduction is quantified through the antenna patterns, $F_{+}$and $F_{\times}$, which always take values less than 1 . As demonstrated in $[55,56]$, after averaging the detector response over both location and orientation, the binary system will reduce the strain recovered by a factor of 2.26 . Thus, this can be used to define the average range of the detector as

$$
D_{\text {avg }}=\frac{D_{\max }}{2.26} .
$$

The average sensitive distance allows us to approximate limits on the coalescence rate from null results for a general GWs search. The loudest event statistic formalism [57] states that we can constrain the binary merger rate for a specific mass bin, $i$, at $90 \%$ confidence level (CL) as

$$
\mathcal{R}_{90, i}=\frac{2.3}{\langle V T\rangle_{i}},
$$

where $\langle V T\rangle_{i}$ is the sensitive volume-time, and is given by

$$
\langle V T\rangle_{i}=\frac{4}{3} \pi D_{a v g, i}^{3} T .
$$

Here, $T$ is the analyzable live-time of the detectors. This method provides an excellent approximation of the sensitive 4-volume. We will use this methodology to estimate the rates in the sub-solar mass region. Similar approach has been applied previously in [5]. We assume $T=1 \mathrm{yr}$, in all our results.

\section{Results}

Figure 2 on the left panel shows the distance (horizon distance) to an optimally oriented, and some equal mass binary in the range [0.2-1.0] $M_{\odot}$ as a function of the SNR obtained using the ET and CE power spectral density noises. The vertical line represents $\rho=8$. Within the perspective of ET sensitivity, we note that the maximum distance for detection, assuming the reference value $\rho=8$, is $0.47 \mathrm{Gpc}, 1.05 \mathrm{Gpc}$ and $1.89 \mathrm{Gpc}$ for compact binaries with equal components mass $0.2 M_{\odot}, 0.5 M_{\odot}$ and $1.0 M_{\odot}$, respectively. For CE sensitivity, we find $1.5 \mathrm{Gpc}, 3.2 \mathrm{Gpc}$ and $5.8 \mathrm{Gpc}$ for equal components mass with $0.2 M_{\odot}, 0.5 M_{\odot}$ and $1.0 M_{\odot}$, respectively. Any other combination between these masses will generate intermediate results to these. In these simulations, we consider $f_{\text {low }}=10 \mathrm{~Hz}$ and $f_{\text {upper }}=f_{I S C O}$. Evidently, the horizon distance for CE is greater than ET, because CE has a greater sensitivity.

Figure 2 on the right panel shows the constraints on the merger rate of equal-mass ultra-compact binaries in the range $[0.2,1.0] M_{\odot}$ for both, ET and CE instruments. Table 1 summarizes the estimates for some particular cases. The results for CE can improve up to 1 order of magnitude of the expected merger rate for ET. We do not take into account possible eccentric orbits effects, which may possibly increase the expected value for $\mathcal{R}_{90}[9,10]$.

Table 1. Estimates of the merger rate of equal-mass compact binaries in the range $[0.2,1.0] M_{\odot}$ for ET and CE instruments.

\begin{tabular}{ccc}
\hline Instrument & Component Mass $\left[\boldsymbol{M}_{\odot}\right]$ & $\mathcal{R}_{\mathbf{9 0}}\left[\mathbf{G p c}^{-\mathbf{3}} \mathbf{y r}^{-\mathbf{1}}\right]$ \\
\hline ET & 0.2 & 53 \\
ET & 0.5 & 4.7 \\
ET & 1.0 & 0.924 \\
CE & 0.2 & 1.829 \\
CE & 0.5 & 0.090 \\
CE & 1.0 & 0.029 \\
\hline
\end{tabular}



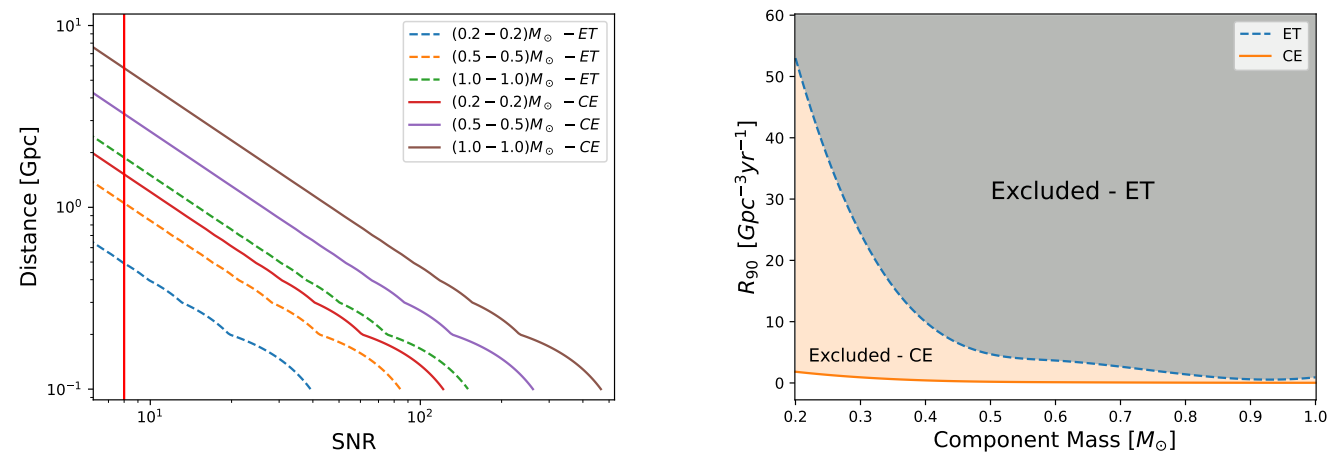

Figure 2. (Left panel): The distance in Gpc units to an optimally oriented, equal mass binary as a function of the signal-to-noise ratio (SNR) for the perspective of the ET and CE sensitivity noise curve. The vertical line represent $S N R \equiv \rho=8$. (Right panel): The merger rate of equal-mass ultra-compact binaries as a function of the components mass in units of $M_{\odot}$.

\subsection{Bounds on Primordial Black Holes}

There is a strong theoretical appeal for the existence of PBHs, especially because $\mathrm{PBHs}$ are dark matter candidates in a broad mass range. Constraint on the binary merger rate places bounds on the total fraction of dark matter made of PBHs, which can be quantified by the parameter $f_{\mathrm{PBH}}$. Several authors have shown possible ways to form PBHs in the early Universe $[15-18,25,58,59]$ and sub-solar mass $\mathrm{PBHs}$ are proposed to exist in various scenarios, for instance, see in [60-62]. From the LIGO/Virgo data, constraints are achieved on sub-solar sources to $<1.0 \times 10^{6} \mathrm{Gpc}^{-3} \mathrm{yr}^{-1}$ and $<1.9 \times 10^{4} \mathrm{Gpc}^{-3} \mathrm{yr}^{-1}$ for $\left(0.2 \mathrm{M}_{\odot}, 0.2 \mathrm{M}_{\odot}\right)$ and $\left(1.0 M_{\odot}, 1.0 M_{\odot}\right)$ ultra-compact binaries [6]. Other analyses to search for sub-solar mass compact-binary mergers in LIGO/VIRGO data that constrain the PBHs populations are presented in [5,8-11]. In particular, see the work in [11] for a summary of the search for non-spinning binary sources, spanning sub-solar mass ranges.

On the other hand, the merger rate of the sub-solar GWs sources is model-dependent, and can depend on different formation scenarios [63-66]. The merger rate assuming a Poisson scenario can be written as

$$
\frac{R_{P B H}(t)}{\mathrm{Gpc}^{-3} \mathrm{yr}^{-1}}=1.6 \times 10^{6} f_{\mathrm{sup}} f_{\mathrm{PBH}}^{53 / 37} \eta^{-34 / 37}\left(\frac{M}{M_{\odot}}\right)^{-32 / 37} \times\left(\frac{t}{t_{0}}\right)^{-34 / 37},
$$

where $f_{\text {sup }}$ is the suppression factor which varies from $10^{-3}$ to 1 [67], $f_{\mathrm{PBH}}$ the fraction of dark matter in the form of PBHs, $\eta$ denotes the reduced mass ratio; $M$ denotes the total mass, $t$ denotes the proper time, and $t_{0}$ denotes the age of the universe today.

We use the MCMC method to analyze the parameters $\theta_{i}=\left\{f_{\text {sup }}, f_{\mathrm{PBH}}\right\}$, building the posterior probability distribution function:

$$
p\left(\theta_{i}, \alpha \mid D\right)=\frac{1}{Z} p(\theta, \alpha) p(D \mid \theta, \alpha),
$$

where $p(\theta, \alpha)$ and $p(D \mid \theta, \alpha)$ are the prior distribution and the likelihood function, respectively. Here, the quantities $D$ and $\alpha$ are the set of observations and possible nuisance parameters. $Z$ is a normalization term. We perform the statistical analysis based on the emcee algorithm [68], assuming the theoretical $R_{P B H}$ model described above with the following uniform priors on the parameters: $f_{\text {sup }} \in\left[10^{-3}, 1\right]$ and $f_{\mathrm{PBH}} \in[0,1]$. During our analysis, we discarded the first $20 \%$ steps of the chain as burn-in. 
Table 2 shows the upper bounds on $f_{\text {sup }}$ and $f_{\text {sup }}$ derived from the expected event rate within the ET sensitivity assuming component mass with $0.2 M_{\odot}, 0.5 M_{\odot}$ and $1.0 M_{\odot}$. Estimates based on CE are one order of magnitude smaller than these. Figure 3 on the left panel shows the parametric space limited to $68 \% \mathrm{CL}$ and $95 \% \mathrm{CL}$ for the case with component mass $0.2 M_{\odot}$. On the right panel, we show the case with component mass $0.5 M_{\odot}$ (label Case I) and $1.0 M_{\odot}($ label Case II).

Table 2. Upper bounds on $f_{\text {sup }}$ and $f_{\mathrm{PBH}}$ (fraction of PBHs in dark matter) derived from the expected event rate within the ET sensitivity.

\begin{tabular}{ccc}
\hline Component Mass $\left[\boldsymbol{M}_{\odot}\right]$ & $f_{\text {sup }}$ & $f_{\text {PBH }}$ \\
\hline 0.2 & $<0.47$ & $<0.022$ \\
0.5 & $<0.43$ & $<7 \times 10^{-3}$ \\
1.0 & $<0.17$ & $<3.4 \times 10^{-3}$ \\
\hline
\end{tabular}
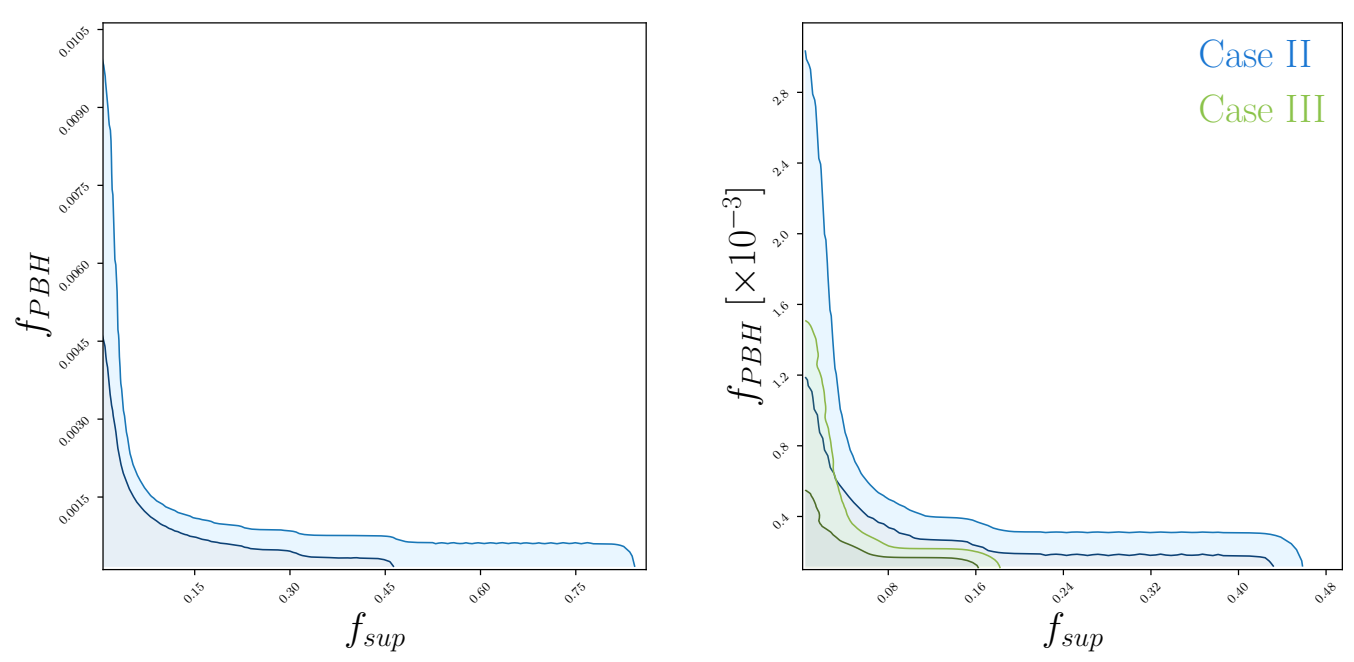

Figure 3. (Left panel): Two-dimensional joint posterior distributions in the $f_{\text {sup }}-f_{P B H}$ plane, with the corresponding $68 \%$ CL and 95\% CL contours, obtained from the expected event rate within the ET sensitivity assuming a binary system with component mass $0.2 M_{\odot}$. (Right panel): Same as in left panel, but for a binary system with equal components mass $0.5 M_{\odot}$ (Case II) and $1.0 M_{\odot}$ (Case III).

It shows that matching the constraints derived from these three mass bins, we can explain $\sim 2.3 \%$ of total dark matter abundance from the ET sensitivity. Using CE, we note $\sim 0.2 \%$ of total dark matter abundance.

Ultra-compact binary system with component mass $0.01 M_{\odot}$ : We repeat the same analysis strategy, but now to verify the feasibility of detecting an ultra-compact binary system with component mass $0.01 M_{\odot}$. Assuming a minimum $\rho=8$, we find that the maximum horizon distances are $\sim 40 \mathrm{Mpc}$ and $\sim 125 \mathrm{Mpc}$ for ET and CE, respectively. The estimates of the merger rate of this compact binaries are $99,034 \mathrm{Gpc}^{-3} \mathrm{yr}^{-1}$ and $3245 \mathrm{Gpc}^{-3} \mathrm{yr}^{-1}$ for ET and $\mathrm{CE}$, respectively. The general upper bounds on $f_{\mathrm{PBH}}$, which can fit these merger rates are $f_{\mathrm{PBH}}<0.68$ and $f_{\mathrm{PBH}}<0.06$ for ET and $\mathrm{CE}$, respectively. We show the parametric space limited to $68 \%$ CL and 95\% CL in Figure 4.

Therefore, within ET perspective, binary system with component mass $0.01 M_{\odot}$, can represent up to $\sim 68 \%$ of dark matter (if $f_{\text {sup }}=10^{-3}$ ). Taking the contributions across the mass range $\left[10^{-2}, 1.0\right] M_{\odot}$ combined, we have $f_{\mathrm{PBH}}<0.70$, that is, a limit of $\sim 70 \%$. These constraints can be significantly improved within CE perspectives, where we note $f_{\mathrm{PBH}}<0.06$, finding a maximum $\sim 6 \%$ for the abundance of dark matter. 


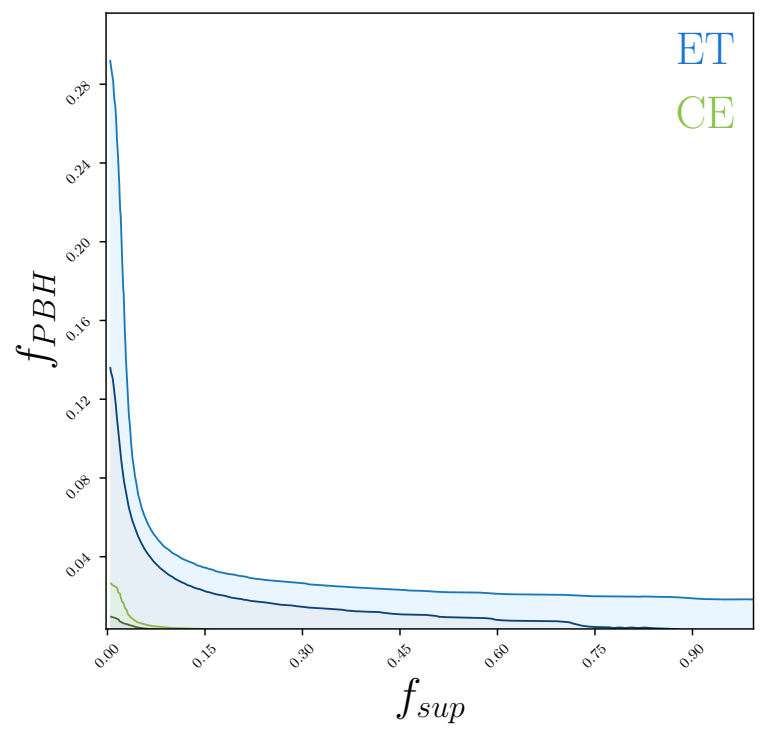

Figure 4. Two-dimensional joint posterior distributions in the $f_{\text {sup }}-f_{\mathrm{PBH}}$ plane, with the corresponding $68 \%$ CL and $95 \%$ CL contours, obtained from the expected event rate within the ET and CE sensitivity assuming a binary system with component mass $0.01 \mathrm{M}_{\odot}$.

It is important to mention that the mass range analyzed in this article, and the upper bound that we derive on the PBHs abundance, are similar to others obtained due to the search using microlensing effects and other observational techniques. If a compact object crosses the line of sight of a star, it may produce a so-called microlensing effect, which implies a transient and achromatic amplification of its flux. The non-detection of these events leads to bounds on the maximum abundance of PBHs about $f_{P B H}<0.01-0.1$ by the MACHO [69] and EROS [70] surveys. In [71], the authors analyze the lack of lensing in type Ia supernovae (from the JLA sample) to constrain the PBH population in the mass range $\left[10^{-2}, 10^{4}\right] M_{\odot}$, about $f_{P B H}<0.35$. We refer to the work in [44] where other PBHs abundance are mentioned in the same mass range used in our research. On the other hand, the significance of our results is that the next generation the GW detectors will be able to detect such events, if they exist, and significantly improve the current bound on $f_{P B H}$ in GWs searchs. Coincidentally, we concluded that these future ground-based GWs detectors like ET and CE, might constraint the abundance of the sub-solar PBHs to similar bound that microlensing effect. Some advantages is that a directly detection by GWs in the future could come to be direct evidence for the existence of such compact objects, and through real data, we can study in detail the whole parameter space of such binary systems, of which can provide more details about its origin and dynamics.

\subsection{Other Alternatives for Sub-Solar-Mass Objects}

It is now clear that it will be possible to detect sub-solar-mass objects with high significance (high $\rho$ value), from $40 \mathrm{Mpc}$ up to a few Gpc distance with ET and CE. See Figure 2 on the left panel for a summary. In addition to interpreting these ultra-compact objects as PHBs, there is a wide range of theoretical predictions, which in principle, lead to the formation of objects with mass below $1 M_{\odot}$.

In [72], the authors proposed a mechanism that can convert a sizable fraction of neutron stars into BHs with mass $\sim 1 M_{\odot}$, too light to be produced via standard stellar evolution. Such BHs could be in binary systems, and thus may be searched by GWs detectors. Furthermore, sub-Chandrasekhar mass BHs may also exist [73], where stellar objects catastrophically accrete non-annihilating dark matter, and the small dark core subsequently collapses, eating up the host star and transmuting it into a BH. Rotating dark stars, constituted for both fermionic and bosonic equations of state, in the presence of self-interacting dark matter, can also generate ultra-compact objects with $<1 M_{\odot}$ (see [74] and references therein). Quark stars [45], anisotropic dark matter stars [75], and many other 
mechanisms can form sub-solar-mass objects (see [45] for a review). Therefore, there is a rich source of models and physics with sub-Chandrasekhar mass, which can not be explained by stellar evolution. These may certainly involve a new physics, and may be alternatives to PBHs. Certainly the mechanism generation of GWs in these systems must be better modeled and understood, to search possible imprints of the such ultracompact objects.

\section{Final Remarks}

We have presented the search (a forecast) for ultracompact binary mergers with components mass below $1 M_{\odot}$ within the expected sensitivity for the ET and CE detectors. We have concluded that ultracompact binary systems with equal component mass of $10^{-2} M_{\odot}$ up to $1 M_{\odot}$ could be detected with high significance since $40 \mathrm{Mpc}(125 \mathrm{Mpc})$, for very low mass system with $10^{-2} M_{\odot}$ in the ET (CE) sensitivity, up to $1.89 \mathrm{Gpc}(5.8 \mathrm{Gpc})$, for binary system with component mass $1 M_{\odot}$, in the ET (CE) sensitivity band, respectively. For possible components mass of the order of magnitude less than $10^{-2}$, within the approach developed here, it will be difficult to have signals with significant SNR values, that is, $\rho>8$.

We have determined the merger rate in the mass range $\left[10^{-2}-1\right] M_{\odot}$, and then constrained the abundance of primordial black holes as a fraction of the total dark matter in this range mass, quantifying $f_{\mathrm{PBH}}<0.70$ and $f_{\mathrm{PBH}}<0.06$, from the perspective of $\mathrm{ET}$ and $\mathrm{CE}$, respectively. Therefore, $\mathrm{CE}$ puts tight constraint on $f_{\mathrm{PBH}}$. Considering non-negligible spin, eccentric orbits, and possible tidal deformability effects on the waveform can improve and bring new perspectives in this regard, as the origin of these systems can have very different physical aspects. On the other hand, still in this generation of observations, the Advanced LIGO/Virgo in their final design sensitivities, will be more sensitive to detect possible mergers of ultra-compact binaries, which may open new trends for new physics involving sub-solar mass ultra-compact objects.

Funding: This research received no external funding.

Data Availability Statement: The data underlying this article will be shared on request to the corresponding author.

Acknowledgments: I am grateful to Jose C. N. de Araujo and Suresh Kumar for very constructive comments and suggestions. I would like to thank the financial support from the Fundação de Amparo à Pesquisa do Estado de São Paulo (FAPESP, São Paulo Research Foundation) under the project No. 2018/18036-5.

Conflicts of Interest: The authors declare no conflict of interest.

\section{References}

1. The LIGO Scientific Collaboration; The Virgo Collaboration. GWTC-2.1: Deep Extended Catalog of Compact Binary Coalescences Observed by LIGO and Virgo During the First Half of the Third Observing Run. arXiv 2021, arXiv:2108.01045.

2. The LIGO Scientific Collaboration; The Virgo Collaboration. GWTC-2: Compact Binary Coalescences Observed by LIGO and Virgo during the First Half of the Third Observing Run. Phys. Rev. X 2021, 11, 021053. [CrossRef]

3. The LIGO Scientific Collaboration; The Virgo Collaboration. Binary Black Hole Mergers in the First Advanced LIGO Observing Run. Phys. Rev. X 2016, 6, 041015. [CrossRef]

4. The LIGO Scientific Collaboration; The Virgo Collaboration. Search for intermediate mass black hole binaries in the first and second observing runs of the Advanced LIGO and Virgo network. Phys. Rev. D 2019, 100, 064064. [CrossRef]

5. Magee, R.; Deutsch, A.S.; McClincy, P.; Hanna, C.; Horst, C.; Meacher, D.; Messick, C.; Shandera, S.; Wade, M. Methods for the detection of gravitational waves from subsolar mass ultracompact binaries. Phys. Rev. D 2018, 98, 103024. [CrossRef]

6. The LIGO Scientific Collaboration; The Virgo Collaboration. Search for Subsolar-Mass Ultracompact Binaries in Advanced LIGO's First Observing Run. Phys. Rev. Lett. 2018, 121, 231103. [CrossRef] [PubMed]

7. The LIGO Scientific Collaboration; The Virgo Collaboration. Search for Subsolar Mass Ultracompact Binaries in Advanced LIGO Second Observing Run. Phys. Rev. Lett. 2019, 123, 161102. [CrossRef]

8. Nitz, A.H.; Wang, Y.F. Search for Gravitational Waves from the Coalescence of Subsolar Mass and Eccentric Compact Binaries. Astrophys. J. 2021, 915, 54. [CrossRef]

9. Nitz, A.H.; Wang, Y.F. Search for gravitational waves from the coalescence of sub-solar mass binaries in the first half of Advanced LIGO and Virgo's third observing run. arXiv 2021, arXiv:2106.08979. 
10. Wang, Y.F.; Nitz, A.H. Prospects for Detecting Gravitational Waves from Eccentric Subsolar Mass Compact Binaries. Astrophys. J. 2021, 912, 53. [CrossRef]

11. Phukon, K.S.; Baltus, G.; Caudill, S.; Clesse, S.; Depasse, A.; Fays, M.; Fong, H.; Kapadia, S.J.; Magee, R.; Tanasijczuk, A.J. The hunt for sub-solar primordial black holes in low mass ratio binaries is open. arXiv 2021, arXiv:2105.11449.

12. Özel, F.; Psaltis, D.; Narayan, R.; McClintock, J.E. The black hole mass distribution in the galaxy. Astrophys. J. 2010, 725, 1918-1927. [CrossRef]

13. Farr, W.M.; Sravan, N.; Cantrell, A.; Kreidberg, L.; Bailyn, C.D.; Mandel, I.; Kalogera, V. The mass distribution of stellar-mass black holes. Astrophys. J. 2011, 741, 103. [CrossRef]

14. Kreidberg, L.; Bailyn, C.D.; Farr, W.M.; Kalogera, V. Mass measurements of black holes in x-ray transients: Is there a mass gap? Astrophys. J. 2012, 757, 36. [CrossRef]

15. Zel'dovich, Y.B.; Novikov, I.D. The Hypothesis of Cores Retarded during Expansion and the Hot Cosmological Model. Sov. Astron. 1967, 10, 602.

16. Hawking, S. Gravitationally collapsed objects of very low mass. Mon. Not. R. Astron. Soc. 1971, 152, 75. [CrossRef]

17. Carr, B.J. The primordial black hole mass spectrum. Astrophys. J. 1975, 201, 1-19. [CrossRef]

18. Khlopov, M.Y.; Polnarev, A.G. Primordial black holes as a cosmological test of grand unification. Phys. Lett. B 1980, 97, 383-387. [CrossRef]

19. Chapline, G.F. Cosmological effects of primordial black holes. Nature 1975, 253, 251-252. [CrossRef]

20. Bird, S.; Cholis, I.; Muñoz, J.B.; Ali-Haïmoud, Y.; Kamionkowski, M.; Kovetz, E.D.; Raccanelli, A.; Riess, A.G. Did LIGO Detect Dark Matter? Phys. Rev. Lett. 2016, 116, 201301 . [CrossRef]

21. Luca, V.D.; Franciolini, G.; Pani, P.; Riotto, A. The evolution of primordial black holes and their final observable spins. J. Cosmol. Astropart. Phys. 2020, 2020, 052. [CrossRef]

22. Villanueva-Domingo, P.; Mena, O.; Palomares-Ruiz, S. A Brief Review on Primordial Black Holes as Dark Matter. Front. Astron. Space Sci. 2021, 8, 681084. [CrossRef]

23. Carr, B.; Kühnel, F.; Sandstad, M. Primordial black holes as dark matter. Phys. Rev. D 2016, 94, 083504. [CrossRef]

24. García-Bellido, J. Massive Primordial Black Holes as Dark Matter and their detection with Gravitational Waves. J. Phys. Conf. Ser. 2017, 840, 012032. [CrossRef]

25. Raidal, M.; Vaskonen, V.; Veermäe, H. Gravitational waves from primordial black hole mergers. J. Cosmol. Astropart. Phys. 2017, 2017, 037. [CrossRef]

26. Wang, S.; Wang, Y.F.; Huang, Q.G.; Li, T.G. Constraints on the Primordial Black Hole Abundance from the First Advanced LIGO Observation Run Using the Stochastic Gravitational-Wave Background. Phys. Rev. Lett. 2018, 120, 191102. [CrossRef]

27. Mandic, V.; Bird, S.; Cholis, I. Stochastic Gravitational-Wave Background due to Primordial Binary Black Hole Mergers. Phys. Rev. Lett. 2016, 117, 201102. [CrossRef] [PubMed]

28. Franciolini, G.; Baibhav, V.; Luca, V.D.; Ng, K.K.Y.; Wong, K.W.K.; Berti, E.; Pani, P.; Riotto, A.; Vitale, S. Quantifying the evidence for primordial black holes in LIGO/Virgo gravitational-wave data. arXiv 2021, arXiv:2105.03349.

29. De Luca, V.; Franciolini, G.; Pani, P.; Riotto, A. Bayesian evidence for both astrophysical and primordial black holes: mapping the GWTC-2 catalog to third-generation detectors. J. Cosmol. Astropart. Phys. 2021, 2021, 003. [CrossRef]

30. Domènech, G.; Lin, C.; Sasaki, M. Gravitational wave constraints on the primordial black hole dominated early universe. J. Cosmol. Astropart. Phys. 2021, 2021, 062. [CrossRef]

31. Khalouei, E.; Ghodsi, H.; Rahvar, S.; Abedi, J. Possibility of primordial black holes as the source of gravitational wave events in the advanced LIGO detector. Phys. Rev. D 2021, 103, 084001. [CrossRef]

32. Wong, K.W.; Franciolini, G.; De Luca, V.; Baibhav, V.; Berti, E.; Pani, P.; Riotto, A. Constraining the primordial black hole scenario with Bayesian inference and machine learning: The GWTC-2 gravitational wave catalog. Phys. Rev. D 2021, 103, 023026. [CrossRef]

33. Hall, A.; Gow, A.D.; Byrnes, C.T. Bayesian analysis of LIGO-Virgo mergers: Primordial versus astrophysical black hole populations. Phys. Rev. D 2020, 102, 123524. [CrossRef]

34. Jedamzik, K. Consistency of Primordial Black Hole Dark Matter with LIGO/Virgo Merger Rates. Phys. Rev. Lett. 2021, 126, 051302. [CrossRef] [PubMed]

35. Lehmann, B.V.; Profumo, S.; Yant, J. Model-independent discovery prospects for primordial black holes at LIGO. Mon. Not. R. Astron. Soc. 2020, 501, 3727-3740 . [CrossRef]

36. Luca, V.D.; Franciolini, G.; Pani, P.; Riotto, A. The Minimum Testable Abundance of Primordial Black Holes at Future GravitationalWave Detectors. arXiv 2021, arXiv:2106.13769.

37. Mukherjee, S.; Silk, J. Can we distinguish astrophysical from primordial black holes via the stochastic gravitational wave background? Mon. Not. R. Astron. Soc. 2021, 506, 3977-3985. [CrossRef]

38. Cui, W.; Huang, F.; Shu, J.; Zhao, Y. Stochastic Gravitational Wave Background from PBH-ABH Mergers. arXiv 2021, arXiv:2108.04279.

39. Bartolo, N.; De Luca, V.; Franciolini, G.; Peloso, M.; Racco, D.; Riotto, A. Testing primordial black holes as dark matter with LISA. Phys. Rev. D 2019, 99, 103521. [CrossRef]

40. Kozaczuk, J.; Lin, T.; Villarama, E. Signals of primordial black holes at gravitational wave interferometers. arXiv 2021, arXiv:2108.12475. 
41. Barsanti, S.; Luca, V.D.; Maselli, A.; Pani, P. Detecting Subsolar-Mass Primordial Black Holes in Extreme Mass-Ratio Inspirals with LISA and Einstein Telescope arXiv 2021, arXiv:2109.02170.

42. Chen, Z.C.; Huang, Q.G. Distinguishing primordial black holes from astrophysical black holes by Einstein Telescope and Cosmic Explorer. J. Cosmol. Astropart. Phys. 2020, 2020, 039. [CrossRef]

43. Ng, K.K.Y.; Chen, S.; Goncharov, B.; Dupletsa, U.; Borhanian, S.; Branchesi, M.; Harms, J.; Maggiore, M.; Sathyaprakash, B.S.; Vitale, S. On the single-event-based identification of primordial black hole mergers at cosmological distances arXiv 2021, arXiv:2108.07276.

44. Carr, B.; Kühnel, F. Primordial Black Holes as Dark Matter: Recent Developments. Annu. Rev. Nucl. Part. Sci. 2020, 70, 355-394. [CrossRef]

45. Cardoso, V.; Pani, P. Testing the nature of dark compact objects: A status report. Living Rev. Relativ. 2019, 22, 4. [CrossRef]

46. Maggiore, M.; Van Den Broeck, C.; Bartolo, N.; Belgacem, E.; Bertacca, D.; Bizouard, M.A.; Branchesi, M.; Clesse, S.; Foffa, S.; Garcia-Bellido, J.; et al. Science case for the Einstein telescope. J. Cosmol. Astropart. Phys. 2020, 2020, 050. [CrossRef]

47. Sathyaprakash, B.S.; Belgacem, E.; Bertacca, D.; Caprini, C.; Cusin, G.; Dirian, Y.; Fan, X.; Figueroa, D.; Foffa, S.; Hall, E.; et al. Cosmology and the Early Universe. arXiv 2019, arXiv:1903.09260.

48. Reitze, D.; Adhikari, R.X.; Ballmer, S.; Barish, B.; Barsotti, L.; Billingsley, G.; Brown, D.A.; Chen, Y.; Coyne, D.; Eisenstein, R.; et al. Cosmic Explorer: The U.S. Contribution to Gravitational-Wave Astronomy beyond LIGO. arXiv 2019, arXiv:1907.04833.

49. Available online: http:/ / www.et-gw.eu/index.php/etsensitivities (accessed on 12 January 2022).

50. Hild, S.; Abernathy, M.; Acernese, F.; Amaro-Seoane, P.; Andersson, N.; Arun, K.; Barone, F.; Barr, B.; Barsuglia, M.; Beker, M.; et al. Sensitivity studies for third-generation gravitational wave observatories. Class. Quantum Gravity 2011, 28, 094013. [CrossRef]

51. Available online: https:/ / cosmicexplorer.org/researchers.html (accessed on 12 January 2022).

52. Chiba, T.; Yokoyama, S. Spin distribution of primordial black holes. Prog. Theor. Exp. Phys. 2017, 2017, 083E01. [CrossRef]

53. Luca, V.D.; Desjacques, V.; Franciolini, G.; Malhotra, A.; Riotto, A. The initial spin probability distribution of primordial black holes. J. Cosmol. Astropart. Phys. 2019, 2019, 018. [CrossRef]

54. Postnov, K.A.; Kuranov, A.G.; Mitichkin, N.A. Spins of black holes in coalescing compact binaries. Phys.-Uspekhi 2019, 62, 1153-1161. [CrossRef]

55. Finn, L.S.; Chernoff, D.F. Observing binary inspiral in gravitational radiation: One interferometer. Phys. Rev. D 1993, 47, 2198-2219. [CrossRef]

56. The LIGO Scientific Collaboration; The Virgo Collaboration. Predictions for the rates of compact binary coalescences observable by ground-based gravitational-wave detectors. Class. Quantum Gravity 2010, 27, 173001. [CrossRef]

57. Biswas, R.; Brady, P.R.; Creighton, J.D.E.; Fairhurst, S. The loudest event statistic: General formulation, properties and applications. Class. Quantum Gravity 2009, 26, 175009. [CrossRef]

58. Clesse, S.; García-Bellido, J. The clustering of massive Primordial Black Holes as Dark Matter: Measuring their mass distribution with advanced LIGO. Phys. Dark Universe 2017, 15, 142-147. [CrossRef]

59. Sasaki, M.; Suyama, T.; Tanaka, T.; Yokoyama, S. Primordial black holes-Perspectives in gravitational wave astronomy. Class. Quantum Gravity 2018, 35, 063001. [CrossRef]

60. Nakamura, T.; Sasaki, M.; Tanaka, T.; Thorne, K.S. Gravitational Waves from Coalescing Black Hole MACHO Binaries. Astrophys. J. 1997, 487, L139-L142. [CrossRef]

61. Ioka, K.; Chiba, T.; Tanaka, T.; Nakamura, T. Black hole binary formation in the expanding universe: Three body problem approximation. Phys. Rev. D 1998, 58, 063003. [CrossRef]

62. Carr, B.; Clesse, S.; García-Bellido, J.; Kühnel, F. Cosmic conundra explained by thermal history and primordial black holes. Phys. Dark Universe 2021, 31, 100755. [CrossRef]

63. Luca, V.D.; Desjacques, V.; Franciolini, G.; Riotto, A. The clustering evolution of primordial black holes. J. Cosmol. Astropart. Phys. 2020, 2020, 028. [CrossRef]

64. Jedamzik, K. Primordial black hole dark matter and the LIGO/Virgo observations. J. Cosmol. Astropart. Phys. 2020, $2020,022$. [CrossRef]

65. Ali-Haïmoud, Y.; Kovetz, E.D.; Kamionkowski, M. Merger rate of primordial black-hole binaries. Phys. Rev. D 2017, 96, 123523. [CrossRef]

66. Gow, A.D.; Byrnes, C.T.; Hall, A.; Peacock, J.A. Primordial black hole merger rates: Distributions for multiple LIGO observables. J. Cosmol. Astropart. Phys. 2020, 2020, 031. [CrossRef]

67. Raidal, M.; Spethmann, C.; Vaskonen, V.; Veermäe, H. Formation and evolution of primordial black hole binaries in the early universe. J. Cosmol. Astropart. Phys. 2019, 2019, 018. [CrossRef]

68. Foreman-Mackey, D.; Hogg, D.W.; Lang, D.; Goodman, J. emcee: The MCMC Hammer. Publ. Astron. Soc. Pac. 2013, 125, 306-312. [CrossRef]

69. Alcock, C.; Allsman, R.A.; Alves, D.R.; Axelrod, T.S.; Becker, A.C.; Bennett, D.P.; Cook, K.H.; Dalal, N.; Drake, A.J.; Freeman, K.C.; et al. MACHO Project Limits on Black Hole Dark Matter in the 1-30 $M_{\odot}$ Range. Astrophys. J. 2001, 550, L169-L172. [CrossRef]

70. Tisserand, P.; Le Guillou, L.; Afonso, C.; Albert, J.N.; Andersen, J.; Ansari, R.; Aubourg, E.; Bareyre, P.; Beaulieu, J.P.; Charlot, X.; et al. Limits on the Macho content of the Galactic Halo from the EROS-2 Survey of the Magellanic Clouds. Astron. Astrophys. 2007, 469, 387-404. [CrossRef]

71. Zumalacárregui, M.; Seljak, U. Limits on Stellar-Mass Compact Objects as Dark Matter from Gravitational Lensing of Type Ia Supernovae. Phys. Rev. Lett. 2018, 121, 141101. [CrossRef] [PubMed] 
72. Kouvaris, C.; Tinyakov, P.; Tytgat, M.H. NonPrimordial Solar Mass Black Holes. Phys. Rev. Lett. 2018, 121, 221102. [CrossRef] [PubMed]

73. Dasgupta, B.; Laha, R.; Ray, A. Low Mass Black Holes from Dark Core Collapse. Phys. Rev. Lett. 2021, 126, 141105. [CrossRef] [PubMed]

74. Maselli, A.; Pnigouras, P.; Nielsen, N.G.; Kouvaris, C.; Kokkotas, K.D. Dark stars: Gravitational and electromagnetic observables. Phys. Rev. D 2017, 96, 023005. [CrossRef]

75. Moraes, P.; Panotopoulos, G.; Lopes, I. Anisotropic dark matter stars. Phys. Rev. D 2021, 103, 084023. [CrossRef] 